\title{
Severe Hypopyon Uveitis in the Right Eye of Ankylosing Spondylitis
}

Fan Wang ${ }^{1}$, MD; Chengde Yang ${ }^{1} \mathbb{D}, \mathrm{MD}, \mathrm{PhD}$; Junna Ye ${ }^{1} \mathbb{D}, \mathrm{MD}, \mathrm{PhD}$, Department of Rheumatology and Immunology, Ruijin Hospital, Shanghai Jiao Tong University School of Medicine, Shanghai, China. Address correspondence to Dr. J. Ye, Department of Rheumatology and Immunology, Shanghai Jiao Tong University School of Medicine Affiliated Ruijin Hospital, No. 197 Ruijin Second Road, Huangpu District, Shanghai 200025, China. Email: yjn0912@qq.com. This study was approved by the Committee of Ruijin Hospital (ID: 2016-62), Shanghai, China. Informed consent was obtained from the recruited subject. This work is supported by National Natural Science Foundation of China (81801592), Shanghai Sailing Program (18YF1414100), Excellent Youth B Project (GCQN-2017-B05), and Innovative Research Team of High-level Local Universities in Shanghai.

The most prevailing extraarticular common complication is eye involvement in patients with ankylosing spondylitis (AS), while it is reported that approximately $25-40 \%$ patients suffer from acute anterior uveitis, which may occur at any time during the disease course ${ }^{1}$.

A 46-year-old man presented with no sight in his right eye for 2 years and was referred to our hospital. Anterior segment photography was done under slit lamp biomicroscopes. It showed that conjunctival congestion with dilated episcleral veins, vascularization in the cornea, and plenty of fibrin and hypopyon were found in the anterior chamber. Thus, severe hypopyon uveitis was diagnosed. The significant cataract in the eye also led to the unclearness of the vitreous and retina. He had a history of AS for more than 20 years. Physical examination showed limited motion of the lumbar spine. HLA-B27 was positive and computed tomography showed fusion of both sacroiliac joints. He had uveitis when diagnosed with AS but did not take his medication regularly. The patient was then treated with infliximab at this visit and was followed up.

Hypopyon is a severe inflammatory reaction characterized
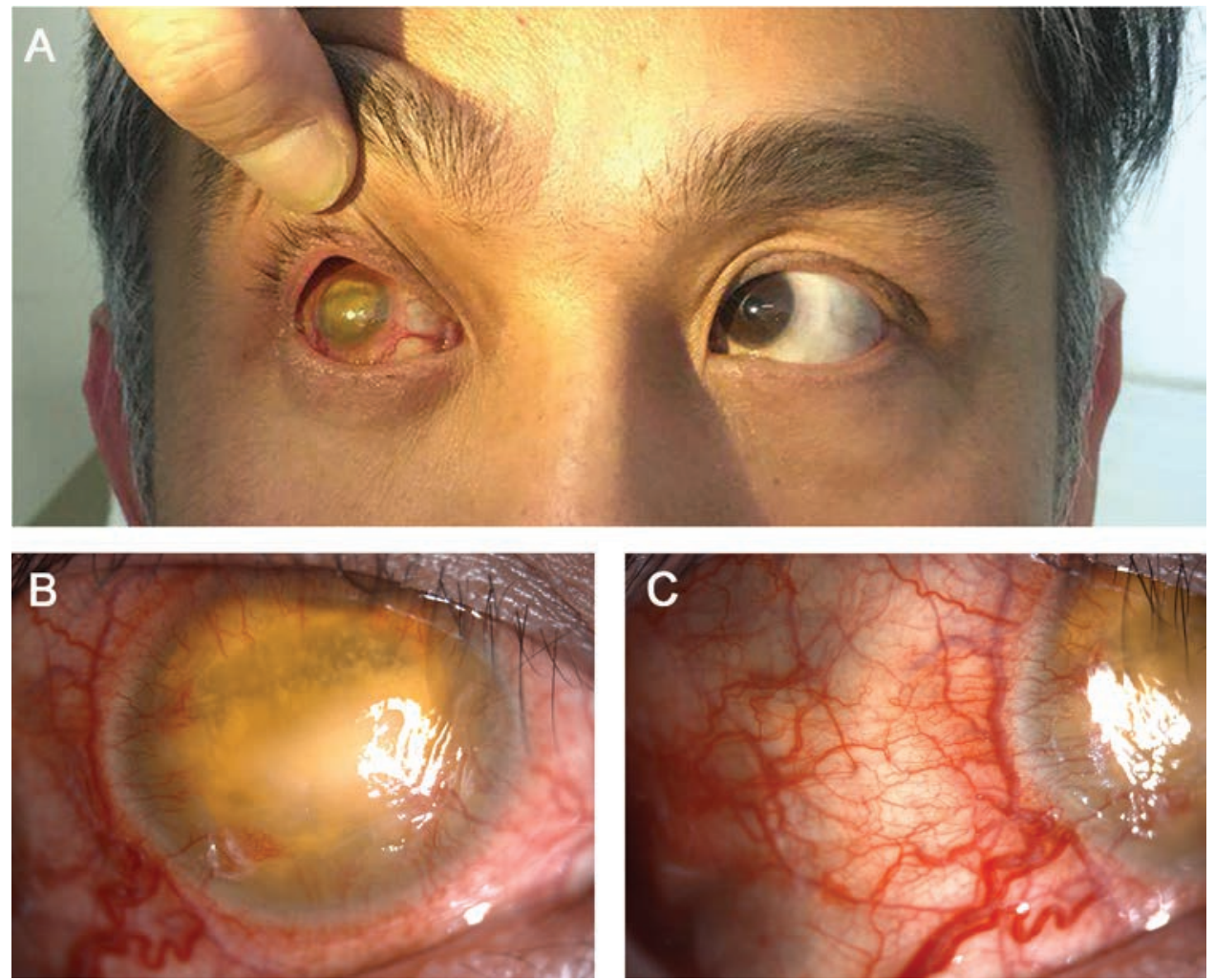

Figure 1. (A) The patient presented with severe hypopyon uveitis that gave rise to no sight in the right eye. (B,C) Anterior segment photography showed that conjunctival congestion with dilated episcleral veins, vascularization in the cornea, and plenty of fibrin and hypopyon were found in the anterior chamber. 
by a layer of white blood cells in the anterior chamber, of which approximately $37 \%$ were considered to be associated with positive HLA-B27 or spondyloarthropathy (HLA-B27-positive or -negative $)^{2}$. Thus, hypopyon will provide clinicians with clues for the diagnosis of a rheumatic disease.

\section{REFERENCES}

1. Maksymowych WP, Chou CT, Russell AS. Matching prevalence of peripheral arthritis and acute anterior uveitis in individuals with ankylosing spondylitis. Ann Rheum Dis 1995;54:128-30.

2. Zaidi AA, Ying GS, Daniel E, Gangaputra S, Rosenbaum JT, Suhler EB, et al; Systemic Immunosuppressive Therapy for Eye Diseases Cohort Study. Hypopyon in Patients with Uveitis. Ophthalmology 2009;117:366-72. 